\title{
How performance of integrated systems of reaction and separation relates to that of parallel and sequential configurations
}

A. L. Paiva, F. X. Malcata

\begin{abstract}
Given the thermodynamic and kinetic limitations which often constrain the extent of chemical reactions and post-reactional separation processes, and therefore constrain the yield and the degree of purity of the resulting products, integration of reaction and separation in a single unit has been under the scope of several $k_{1}$ bioengineering researchers in recent years.

It is the aim of this work to compare the performance of $k_{-1}$ a cascade of $N$ reactor/separator sets, either in series or in parallel, with that of an integrated reaction/separation unit. In order to do so, a Michaelis-Menten reaction in dilute substrate solutions (i.e. a pseudo first order reaction) was considered to take place in either configuration and, under the same reaction and separation conditions, $\quad \bar{n}$ comparison of the performance and efficiency of these configurations was made in terms of fractional recovery of $N$ pure product, total time required to achieve such recovery and rate of recovery.

It was concluded that: (i) the series combination of $r$ reactor/separator sets yields better results, both in terms of $t$ fractional amount of product recovered and time required to do so, than the parallel combination; and (ii) the integrated approach is much more time- and cost-effective than plain cascading, thus making it very attractive from an economic point of view.

$\begin{array}{ll}\text { List of symbols } \\ A & \text { Interfacial area of the separator }\left(\mathrm{m}^{2}\right) \\ C & \text { Molar concentration }\left(\mathrm{mol} \mathrm{m} \mathrm{m}^{-3}\right) \\ i & \begin{array}{l}\text { Generic } i \text {-th reactor/separator unit } \\ (i=1,2, \ldots, N)\end{array} \\ k_{\mathrm{cat}, \mathrm{f}} & \begin{array}{l}\text { first order intrinsic kinetic constant for the } \\ \text { forward reaction }\left(\mathrm{s}^{-1}\right)\end{array} \\ k_{\mathrm{cat}, \mathrm{r}} & \begin{array}{l}\text { first order intrinsic kinetic constant for the } \\ \text { reverse reaction }\left(\mathrm{s}^{-1}\right)\end{array} \\ k_{\mathrm{mt}} & \text { Mass transfer coefficient }\left(\mathrm{m} \mathrm{s}^{-1}\right)\end{array}$
\end{abstract}

A.L. Paiva, F.X. Malcata ( $₫)$

Escola Superior de Biotecnologia, Universidade Católica Portuguesa, P-4200-072 Porto, Portugal

The authors would like to acknowledge financial support provided by JNICT through programs CIENCIA (grant BD/2081/ 92-IF, PhD fellowship) and PRAXIS XXI (grant BD/5568/95, PhD fellowship; and project 2/2.1/BIO/34/94 - Extractive Biocatalysis, research grant).

\author{
$K_{\text {eq }} \quad$ Equilibrium constant of the reaction (-) \\ $K_{\mathrm{m}, \mathrm{S}} \quad$ Dissociation constant of the enzyme/sub- \\ strate complex $\left(\mathrm{mol} \mathrm{m}^{-3}\right)$ \\ $K_{\mathrm{m}, \mathrm{P}} \quad$ Dissociation constant of the enzyme/product \\ complex $\left(\mathrm{mol} \mathrm{m}^{-3}\right)$ \\ Lumped first order rate constant in the \\ forward direction $\left(\mathrm{s}^{-1}\right)$ \\ Lumped first order rate constant in the \\ reverse direction $\left(\mathrm{s}^{-1}\right)$ \\ Number of moles (mol) \\ Number of moles at the outlet stream of the \\ separator constituted by a mixture of $R$ and \\ $\mathrm{P}$ (mol) \\ Number of moles at the outlet stream of the \\ separator constituted by pure $\mathrm{P}$ ( $\mathrm{mol}$ ) \\ Total number of reactor/separator sets \\ Substrate \\ Product \\ Reaction rate $\left(\mathrm{mol} \mathrm{m} \mathrm{m}^{-3} \mathrm{~s}^{-1}\right)$ \\ Time (s) \\ Molar volume $\left(\mathrm{m}^{3} \mathrm{~mol}^{-1}\right)$ \\ Volume of the reactor $\left(\mathrm{m}^{3}\right)$ \\ maximum rate of reaction in the forward \\ reaction $\left(\mathrm{mol} \mathrm{m}^{-3} \mathrm{~s}^{-1}\right)$ \\ maximum rate of reaction in the reverse \\ reaction $\left(\mathrm{mol} \mathrm{m}^{-3} \mathrm{~s}^{-1}\right)$ \\ $k_{\text {cat, } r} \quad$ first order intrinsic kinetic constant for the \\ reverse reaction $\left(s^{-1}\right)$
}

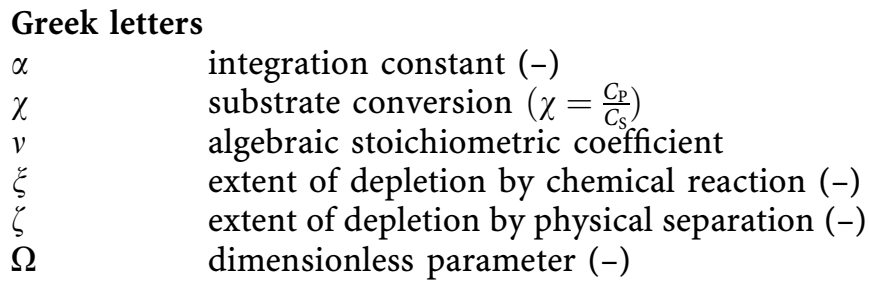

$\begin{array}{ll}\begin{array}{ll}\text { Subscripts } \\ \text { eq }\end{array} & \begin{array}{l}\text { equilibrium conditions } \\ \text { enzyme } \\ \mathrm{E}\end{array} \\ i & \text { generic reactor }(i=1,2, \ldots, N) \\ \mathrm{L} & \text { liquid phase } \\ \mathrm{P} & \text { product } \\ \text { par } & \text { parallel combination } \\ \text { rxn } & \text { reaction } \\ \mathrm{S} & \text { substrate } \\ \text { ser } & \text { series combination } \\ \text { spn } & \text { separation } \\ \text { tot } & \text { total }\end{array}$


tot, $N$

0

\section{Superscripts}

dimensionless variable

1

\section{Introduction}

Transformation of substrate(s) into the corresponding product(s) is the aim of any reactional process. However, owing to thermodynamic and/or kinetic constraints, the yield and purity of the product(s) at the outlet of the reactor does not always attain the levels desired and, for this reason, biochemical reactors have been traditionally followed by separation units, designed in attempts to maximize such levels. Since the costs associated with post-reactional processing of biochemicals are usually high, alternative approaches, such as cascading sets of reactors/separators, have also been proposed and employed by many researchers when trying to improve the processing performance. Given that such analyses generally focus only on sets of reactors/separators used in sequential fashion without considering the hypothesis of their parallel use, one major goal of this work was, thus, to study whether cascading reactor/separator sets in parallel does (or does not) provide better results than in series.

On the other hand, attempts to alleviate limiting factors, such as the high product inhibition and low volumetric productivity that are typical of biochemical processes, have led in the last two decades to comprehensive studies of integrated systems rather than sequential ones [1-7]. Integration can be seen as the limiting situation of cascading when the total number of sets tends to infinite, and entails the possibility of continuously (and immediately) removing the product(s) formed during reaction and, consequently, improving effectiveness of separation since bulk concentrations are not allowed to build up. Such practice is, therefore, expected to reduce the aforementioned costs usually associated with post-reaction separation. Examples of integrated approaches which have recently been selected for biochemical processes encompass (but are not limited to) integrated liquid-liquid systems [8-10], integrated vapor-liquid systems [11-17], integrated supercritical fluid systems $[18,19]$, integrated solid-liquid systems [20-28] and integrated solid-gas systems [29-32]. A comprehensive review of applications of integration in biochemical processes has been provided elsewhere [33].

Laane et al. [1] and Tramper et al. [2] have claimed that reaction coupled with in situ separation brings about kinetic enhancements in the case of biochemical processes. However, such claim tends only to consider the point of view of the reaction rather than that of the overall process, constituted by both reaction and separation. Paiva and Malcata [4] have later demonstrated that integration of reaction and separation does not provide a true thermodynamic enhancement if Gibbs' free energy is used as quantitative measure because of its nature of state function (i.e. with changes that are independent of path).
However, it was also demonstrated that, once physical separation is achieved on the molecular level right upon chemical reaction has taken place, integration decreases kinetic limitations via prevention of bulk mixing of product with (unreacted) substrate [6]. In spite of the complexity associated with modelling and prediction of behaviour in the case of integrated approaches, the actual decrease in the total manufacture cost of the product arising from lower reaction times (and thus lower capital investments in smaller reactors able to effect a given conversion of substrate) coupled with separation to a higher extent (and thus lower separation costs in a posteriori less intensive separation processes) may overcome this drawback.

In order to maintain the analysis mathematically tractable throughout this study, the limiting first order behaviour of a generic enzyme-catalyzed reaction was considered. The model system selected consists in a reaction that follows a 1:1 stoichiometry, which is in agreement with the current trend of bioprocess intensification brought about by increasing substrate concentration to the highest degree possible (which also avoids use of solvents that add to downstream separation problems). The performance of a cascade of $N$ similar reactor/separator sets (both in series and in parallel) was then compared with that of an integrated reaction/separation unit. Such comparison was made both in terms of the fractional amount of pure product recovered, its rate of recovery and total time (time of reaction plus time of separation) required to achieve the same extent of conversion and separation.

\section{2}

\section{Mathematical development}

Let us consider a chemical transformation of substrate (S) into product $(\mathrm{P})$ occuring via an enzyme-catalyzed reaction according to the following Michaelis-Menten reversible mechanism assumed to satisfy quasi-equilibrium conditions at all times:

$\mathrm{E}+\mathrm{S} \stackrel{K_{\mathrm{m}, \mathrm{s}}}{\rightleftarrows} \mathrm{ES} \underset{k_{\text {cat }, \mathrm{r}}}{\stackrel{k_{\mathrm{cat}, \mathrm{f}}}{\rightleftarrows}} \mathrm{EP} \stackrel{K_{\mathrm{m}, \mathrm{P}}}{\rightleftarrows} \mathrm{E}+\mathrm{P}$,

where $K_{\mathrm{m}, \mathrm{S}}$ and $K_{\mathrm{m}, \mathrm{P}}$ are the dissociation constants of the enzyme/substrate complex and the enzyme/product complex, respectively, and $k_{\mathrm{cat}, \mathrm{f}}$ and $k_{\mathrm{cat}, \mathrm{r}}$ are first order, intrinsic kinetic constants for the forward and reverse reaction, respectively. Under this postulated mechanism, the following rate expression can be derived [34]:

$r=\frac{v_{\max , \mathrm{f}} \frac{C_{\mathrm{S}}}{K_{\mathrm{m}, \mathrm{s}}}-v_{\max , \mathrm{r}} \frac{C_{\mathrm{P}}}{K_{\mathrm{m}, \mathrm{P}}}}{1+\frac{C_{\mathrm{S}}}{K_{\mathrm{m}, \mathrm{S}}}+\frac{C_{\mathrm{p}}}{K_{\mathrm{m}, \mathrm{P}}}}$,

where $r$ denotes the reaction rate, $C_{\mathrm{S}}$ and $C_{\mathrm{P}}$ represent the molar concentration of substrate and product, respectively, and $v_{\text {max.f }}$ and $v_{\text {max.r }}$ represent the maximum rates of reaction in the forward and reverse direction under saturation conditions of enzyme, and are given by:

$$
\begin{aligned}
& v_{\mathrm{max}, \mathrm{f}}=k_{\mathrm{cat}, \mathrm{f}} C_{\mathrm{E}, \mathrm{tot}}, \\
& v_{\mathrm{max}, \mathrm{r}}=k_{\mathrm{cat}, \mathrm{r}} C_{\mathrm{E}, \mathrm{tot}},
\end{aligned}
$$


where $C_{\mathrm{E}, \text { tot }}$ denotes the total concentration of catalytic sites; if the enzyme contains only one catalytic site per molecule, then $C_{\mathrm{E}, \text { tot }}$ is equal to the total concentration of enzyme.

If we now assume that both the concentrations of substrate and product are very small (and remain as such) when compared with the Michaelis-Menten parameters $K_{\mathrm{m}, \mathrm{S}}$ and $K_{\mathrm{m}, \mathrm{P}}$, respectively, then their contribution to the denominator of Eq. (2) is negligible; this equation may thus be simplified to:

$r=k_{1} C_{\mathrm{S}}-k_{-1} C_{\mathrm{P}}$,

which is typical of first order reversible reactions, where $k_{1}$ and $k_{-1}$ can be viewed as lumped first order rate constants defined as $v_{\text {max, }} / K_{\mathrm{m}, \mathrm{S}}$ and $v_{\text {max }, \mathrm{r}} / K_{\mathrm{m}, \mathrm{P}}$, respectively.

One necessary condition for chemical equilibrium corresponds to $r=0$ which, in view of Eq. (4), implies that:

$k_{1} C_{\mathrm{S}, \mathrm{eq}}=k_{-1} C_{\mathrm{P}, \mathrm{eq}}$,

where subscript eq denotes equilibrium conditions; upon algebraic rearrangement, Eq. (5) can take the form:

$\frac{k_{1}}{k_{-1}}=\frac{C_{\mathrm{P}, \mathrm{eq}}}{C_{\mathrm{S}, \mathrm{eq}}}=K_{\mathrm{eq}}$,

where $K_{\mathrm{eq}}$ is the equilibrium constant of the reaction.

Combination of Eqs. (4) and (6) then gives:

$r=k_{1}\left(C_{\mathrm{S}}-\frac{C_{\mathrm{P}}}{K_{\mathrm{eq}}}\right)$.

In view of the stoichiometry apparent in Eq. (1), one can also write:

$C_{\mathrm{S}, 0}+C_{\mathrm{P}, 0}=C_{\mathrm{S}}+C_{\mathrm{P}}$

where subscript 0 denotes initial conditions; if, as usual, no products are initially present (i.e. $C_{\mathrm{P}, 0}=0$ ), then, at any time, Eq. (8) becomes:

$C_{\mathrm{P}}=C_{\mathrm{S}, 0}-C_{\mathrm{S}}$.

Combination of Eqs. (7) and (9) yields:

$r=k_{1}\left(1+\frac{1}{K_{\mathrm{eq}}}\right) C_{\mathrm{S}}-\frac{k_{1} C_{\mathrm{S}, 0}}{K_{\mathrm{eq}}}$.

Consider now a batch stirred tank reactor (which will eventually be a part of a combination of multiple identical reactors) where the aforementioned biochemical reaction is brought about. The mass balance to substrate $S$ in said reactor takes the form:

$\left(-v_{\mathrm{S}}\right) V_{i} r\left\{C_{\mathrm{S}}\right\}+\frac{\mathrm{d} n_{\mathrm{S}}}{\mathrm{d} t}=0$,

where $v_{\mathrm{S}}$ denotes the algebraic stoichiometric coefficient of substrate $S$ (equal to -1 in our case), $V$ denotes the

(useful) volume of a reactor, subscript $i$ refers to the $i$-th reactor, $n_{\mathrm{S}}$ denotes the number of moles of $\mathrm{S}$ and $t$ denotes the time of reaction. Using Eq. (10) in Eq. (11), one gets:

$\frac{\mathrm{d} n_{\mathrm{S}}}{\mathrm{d} t}=\frac{k_{1} V_{i} C_{\mathrm{S}, 0}}{K_{\mathrm{eq}}}-V_{i} k_{1}\left(1+\frac{1}{K_{\mathrm{eq}}}\right) C_{\mathrm{S}}$.

Recalling the definition of molar concentration (i.e. $C=n / V$ ), Eq. (12) can be transformed into: $\frac{\mathrm{d} n_{\mathrm{S}}}{\mathrm{d} t}=\frac{k_{1} n_{\mathrm{S}, 0}}{K_{\mathrm{eq}}} \frac{V_{i}}{V_{0}}-k_{1}\left(1+\frac{1}{K_{\mathrm{eq}}}\right) n_{\mathrm{S}}$.

Furthermore, under the assumption that the mixture of $S$ and $\mathrm{P}$ behaves ideally from a thermodynamic point of view, then:

$v=\frac{V_{i}}{n_{i}}=\frac{V_{0}}{n_{\mathrm{S}, 0}}$,

where $v$ denotes the molar volume of either species and $n_{i}$ the total number of moles of both compounds in the $i$-th reactor (which remains constant between inlet and outlet of the reactor). Substitution of Eq. (14) in Eq. (13) followed by integration via separation of variables gives:

$k_{1}\left(1+\frac{1}{K_{\mathrm{eq}}}\right) t=\alpha-\ln \left\{\frac{k_{1}}{K_{\mathrm{eq}}} n_{i}-k_{1}\left(1+\frac{1}{K_{\mathrm{eq}}}\right) n_{\mathrm{S}}\right\}$,

where $\alpha$ is an integration constant which can be calculated via a suitable initial condition; one such condition is given by:

$n_{\mathrm{S}}\{t=0\}=n_{\mathrm{S}, i-1}$,

where subscript $i-1$ denotes inlet to the $i$-th reactor.

Upon combination of Eqs. (15) and (16), one is led to:

$k_{1}\left(1+\frac{1}{K_{\mathrm{eq}}}\right) t=\ln \left\{\frac{k_{1}\left(1+\frac{1}{K_{\mathrm{eq}}}\right) n_{\mathrm{S}, i-1}-\frac{k_{1}}{K_{\mathrm{eq}}} n_{i}}{k_{1}\left(1+\frac{1}{K_{\mathrm{eq}}}\right) n_{\mathrm{S}}-\frac{k_{1}}{K_{\mathrm{eq}}} n_{i}}\right\}$,

where $t$ and $n_{\mathrm{S}}$ denote generic time and number of moles of substrate, respectively.

In a way similar to the introduction of Eq. (16), Eq. (17) can now be rearranged to give:

$t_{\mathrm{rxn}, i}=\frac{K_{\mathrm{eq}}}{k_{1}\left(1+K_{\mathrm{eq}}\right)} \ln \left\{\frac{\left(1+K_{\mathrm{eq}}\right) n_{\mathrm{S}, i-1}-n_{i}}{\left(1+K_{\mathrm{eq}}\right) n_{\mathrm{S}, i}-n_{i}}\right\}$,

where subscript rxn refers to a reaction unit (in this case the $i$-th reaction unit) and where the inlet and outlet number of moles of substrate are denoted by $n_{\mathrm{S}, i-1}$ and $n_{\mathrm{S}, i}$, respectively. If a given extent of depletion by chemical reaction, $\xi$, is to be ensured, viz.:

$\xi=\frac{n_{\mathrm{S}, i}}{n_{\mathrm{S}, i-1}}$,

then Eq. (18) can be rewritten in dimensionless form as:

$t_{\mathrm{rxn}, i}^{*}=\frac{K_{\mathrm{eq}}}{\left(1+K_{\mathrm{eq}}\right)} \ln \left\{\frac{\left(1+K_{\mathrm{eq}}\right) n_{\mathrm{S}, i-1}^{*}-n_{i}^{*}}{\left(1+K_{\mathrm{eq}}\right) \xi n_{\mathrm{S}, i-1}^{*}-n_{i}^{*}}\right\}$,

provided that the following dimensionless variables are introduced:

$t^{*}=k_{1} t$

$n_{\mathrm{S}}^{*}=\frac{n_{\mathrm{S}}}{n_{\mathrm{S}, 0}}$,

$n_{i}^{*}=\frac{n_{i}}{n_{0}}$. 
Consider now a batch stirred tank separator (which will eventually be a part of a combination of multiple identical separators) where the aforementioned physical separation of a fraction of (pure) $\mathrm{P}$ from the reaction stream is brought about. The mass balance to product $\mathrm{P}$ in said separator takes the form:

$k_{\mathrm{mt}} A C_{\mathrm{P}}+\frac{\mathrm{d} n_{\mathrm{P}}}{\mathrm{d} t}=0 ，$

where $A$ denotes the (constant) interfacial area of a separator, $n_{\mathrm{P}}$ denotes the number of moles of $\mathrm{P}, k_{\mathrm{mt}}$ denotes the separation constant and $t$ denotes, in this case, the time of separation. Recalling the definition of molar concentration (i.e. $C=n / V$ ) coupled again with the assumption that the mixture of $\mathrm{S}$ and $\mathrm{P}$ behaves ideally from a thermodynamic point of view, and recalling Eq. (14), Eq. (24) then becomes:

$\frac{\mathrm{d} n_{\mathrm{P}}}{\mathrm{d} t}=-\frac{k_{\mathrm{mt}} A}{v} \frac{n_{\mathrm{P}}}{n_{\mathrm{P}}+n_{\mathrm{S}}}$,

which can be rearranged to give:

$\frac{\mathrm{d}\left(n_{\mathrm{P}}+n_{\mathrm{S}}\right)}{\mathrm{d} t}=-\frac{k_{\mathrm{mt}} A}{v} \frac{\left(n_{\mathrm{P}}+n_{\mathrm{S}}\right)-n_{\mathrm{S}}}{n_{\mathrm{P}}+n_{\mathrm{S}}}$,

where $n_{\mathrm{S}}$ is in fact a constant for the purpose of partial integration with respect to $\left(n_{\mathrm{P}}+n_{\mathrm{S}}\right)$ once it is assumed that no substrate will leave the separator in the outlet stream of pure P. Integration of Eq. (26) via separation of variables gives:

$\frac{k_{\mathrm{mt}} A}{v} t=\alpha-n_{\mathrm{P}}-n_{\mathrm{S}} \ln \left\{n_{\mathrm{P}}\right\}$,

where $\alpha$ is an integration constant which can be calculated via a suitable initial condition; one such condition is given by:

$n_{\mathrm{P}}\{t=0\}=n_{\mathrm{P}, i} ; \quad n_{\mathrm{S}}\{t=0\}=n_{\mathrm{S}, i}$,

where subscript $i$ denotes outlet from the $i$-th reactor and, therefore, inlet of the $i$-th separator. Upon combination of Eqs. (27) and (28), one is led to:

$\frac{k_{\mathrm{mt}} A}{v} t=n_{\mathrm{P}, i}-n_{\mathrm{P}}+\left(n_{\mathrm{S}, i} \ln \left\{n_{\mathrm{P}, i}\right\}-n_{\mathrm{S}} \ln \left\{n_{\mathrm{P}}\right\}\right)$,

where $t, n_{\mathrm{S}}$ and $n_{\mathrm{P}}$ denote generic time, and number of moles of substrate and product, respectively.

In a way similar to the introduction of Eq. (28), Eq. (29) can be rearranged to give:

$\frac{k_{\mathrm{mt}} A}{v} t_{\mathrm{spn}, i}=n_{\mathrm{P}, i}-\hat{n}_{\mathrm{P}, i}+n_{\mathrm{S}, i} \ln \left\{\frac{n_{\mathrm{P}, i}}{\hat{n}_{\mathrm{P}, i}}\right\}$,

where subscript spn refers to a separation unit and the circumflex ${ }^{\wedge}$ refers to the outlet stream from the separator constituted by a mixture of $\mathrm{R}$ and $\mathrm{P}$. If a given extent of depletion by physical separation, $\zeta$, is to be ensured, viz.:

$\zeta=\frac{\hat{n}_{\mathrm{P}, i}}{n_{\mathrm{P}, i}}$,

then Eq. (31) can be rewritten in dimensionless form as: $t_{\mathrm{spn}, i}^{*}=\Omega\left(n_{\mathrm{P}, i}^{*}(1-\zeta)+n_{\mathrm{S}, i}^{*} \ln \left\{\frac{1}{\zeta}\right\}\right)$,

provided that the following dimensionless variable and parameter are introduced:

$n_{\mathrm{P}}^{*}=\frac{n_{\mathrm{P}}}{n_{\mathrm{S}, 0}}$,

$\Omega=\frac{v k_{1} n_{\mathrm{S}, 0}}{k_{\mathrm{mt}} A}$,

where parameter $\Omega$ can be viewed as the ratio of two time scales, i.e. $\left(v n_{\mathrm{S}, 0} / k_{\mathrm{mt}} A\right)$ or the time scale associated with molecular transport of $\mathrm{P}$, and $\left(\frac{1}{k_{1}}\right)$ or the time scale associated with chemical reaction. From combination of Eqs. (19) and (22), coupled with the assumption that no $\mathrm{P}$ is present in the inlet stream to the first reactor, one obtains:

$n_{\mathrm{S}, i}^{*}=\xi n_{\mathrm{S}, i-1}^{*}$,

$n_{\mathrm{S}, 0}^{*}=1$.

On the other hand, a material balance to $\mathrm{P}$ in the $i$-th reactor coupled with Eqs. (19), (22), (33) and (35) yields:

$n_{\mathrm{P}, i}^{*}=(1-\xi) n_{\mathrm{S}, i-1}^{*}+\hat{n}_{\mathrm{P}, i-1}^{*}$.

Using Eqs. (31) and (33) in Eq. (36), then:

$\hat{n}_{\mathrm{P}, i}^{*}=\zeta\left((1-\xi) n_{\mathrm{S}, i-1}^{*}+\hat{n}_{\mathrm{P}, i-1}^{*}\right)$,

$\hat{n}_{\mathrm{P}, 0}^{*}=0$,

whereas, considering again that no product is present at the inlet of the first reactor, a material balance to $\mathrm{P}$ in the $i$-th separator combined with Eqs. (33) and (37) gives:

$\bar{n}_{\mathrm{P}, i}^{*}=(1-\zeta)\left((1-\xi) n_{\mathrm{S}, i-1}^{*}+\hat{n}_{\mathrm{P}, i-1}^{*}\right)$,

where the upper $\mathrm{bar}^{-}$refers to the outlet stream of pure $\mathrm{P}$ from the separator.

From combination of Eqs. (25), (37) and (38), and recalling that $n_{i}=n_{\mathrm{S}, i}+n_{\mathrm{P}, i}$, one finally gets:

$n_{i}^{*}=n_{\mathrm{S}, i-1}^{*}+\hat{n}_{\mathrm{P}, i-1}^{*}$.

In view of Eq. (39), Eq. (20) can now be rewritten as:

$t_{\mathrm{rxn}, i}^{*}=\frac{K_{\mathrm{eq}}}{1+K_{\mathrm{eq}}} \ln \left\{\frac{K_{\mathrm{eq}} n_{\mathrm{S}, i-1}^{*}-\hat{n}_{\mathrm{P}, i-1}^{*}}{\left[\left(1+K_{\mathrm{eq}}\right) \xi-1\right] n_{\mathrm{S}, i-1}^{*}-\hat{n}_{\mathrm{P}, i-1}^{*}}\right\}$,

whereas Eq. (32) may, in view of Eqs. (35) and (36), be rewritten as:

$$
\begin{aligned}
t_{\mathrm{spn}, i}^{*}=\Omega & ((1-\xi)(1-\zeta) \\
& \left.-\xi \ln \{\zeta\}) n_{\mathrm{S}, i-1}^{*}+(1-\zeta) \hat{n}_{\mathrm{P}, i-1}^{*}\right) .
\end{aligned}
$$

\section{1}

\section{Series combination}

In the case of a series combination of reactor/separator sets (Fig. 1), then the sequential application of Eq. (35) gives

$n_{\mathrm{S}, i}^{*}=\xi^{i}$, 


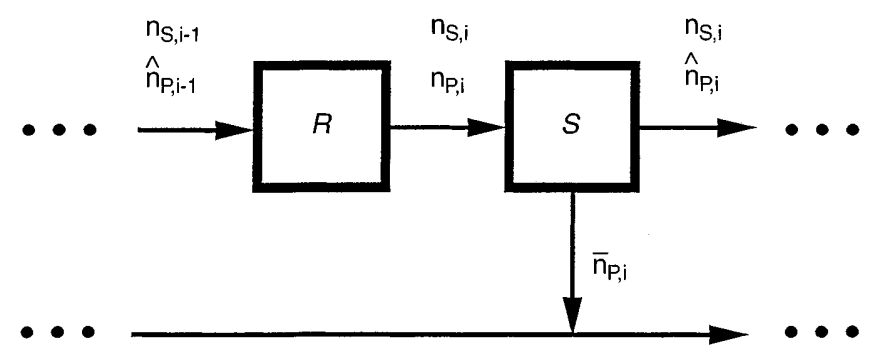

Fig. 1. Schematic representation of the generic $i$-th set, constituted by one reaction (R) and one separation unit (S), in a cascade of $N$ sets in series

whereas sequential combination of Eqs. (35) and (37) gives:

$\hat{n}_{\mathrm{P}, i}^{*}=(1-\xi) \sum_{j=0}^{i-1} \xi^{j} \zeta^{i-j}$.

Furthermore, sequential combination of Eqs. (36), (42) and (43) gives:

$n_{\mathrm{P}, i}^{*}=(1-\xi) \xi^{i-1}\left(1+\sum_{j=0}^{i-2}\left(\frac{\zeta}{\xi}\right)^{i-j-1}\right)$,

whereas sequential combination of Eqs. (38), (42) and (43) gives:

$\bar{n}_{\mathrm{P}, i}^{*}=(1-\zeta)(1-\xi) \xi^{i-1}\left(1+\sum_{j=0}^{i-2}\left(\frac{\zeta}{\xi}\right)^{i-j-1}\right)$,

and sequential combination of Eqs. (39), (42) and (43) gives:

$n_{i}^{*}=\xi^{i-1}\left(1+(1-\xi) \sum_{j=0}^{i-2}\left(\frac{\zeta}{\xi}\right)^{i-j-1}\right)$.

The dimensionless total number of moles of product $\mathrm{P}$ obtained in the $N$ reactors will be given via consideration of Eq. (45), viz.:

$$
\begin{aligned}
\bar{n}_{\mathrm{P}, \mathrm{tot}, N, \mathrm{ser}}^{*} & =\sum_{i=1}^{N} \bar{n}_{\mathrm{P}, i}^{*}=(1-\zeta) \\
& \times\left\{\left(1-\xi^{N}\right)+(1-\xi) \sum_{i=1}^{N} \sum_{j=0}^{i-2}\left(\zeta^{i-j-1} \xi^{j}\right)\right\},
\end{aligned}
$$

where advantage was taken from the formula of summation of the terms of a geometrical series. Subscript tot, $N$, ser refers to the total systems of reactor/separator sets at $i=1,2, \ldots, N$ placed in series. On the other hand, combination of Eqs. (20), (42) and (46) yields:

$$
\begin{aligned}
t_{\mathrm{rxn}, i}^{*} & =\frac{K_{\mathrm{eq}}}{1+K_{\mathrm{eq}}} \\
\times & \times \ln \left\{\frac{K_{\mathrm{eq}}-(1-\xi) \sum_{j=0}^{i-2}\left(\frac{\zeta}{\xi}\right)^{i-j-1}}{\xi\left(1+K_{\mathrm{eq}}\right)-\left[1+(1-\xi) \sum_{j=0}^{i-2}\left(\frac{\xi}{\zeta}\right)^{i-j-1}\right]}\right\} .
\end{aligned}
$$

By a similar token, combination of Eqs. (32), (42) and (44) yields:

$$
=\Omega \xi^{i}\left(\frac{(1-\zeta)(1-\xi)}{\xi}\left(1+\sum_{j=0}^{t_{\mathrm{spn}, i}^{*}}\left(\frac{\zeta}{\xi}\right)^{i-j-1}\right)+\ln \left\{\frac{1}{\zeta}\right\}\right) .
$$

The total dimensionless reaction time can be obtained via sequential combination of Eq. (48), viz.:

$$
\begin{aligned}
& t_{\mathrm{rxn}, \mathrm{tot}, N, \mathrm{ser}}^{*}=\sum_{i=1}^{N} t_{\mathrm{rxn}, i}^{*}=\frac{K_{\mathrm{eq}}}{1+K_{\mathrm{eq}}} \\
& \times \ln \left\{\prod_{i=1}^{N}\left\{\frac{K_{\mathrm{eq}}-(1-\xi) \sum_{j=0}^{i-2}\left(\frac{\zeta}{\xi}\right)^{i-j-1}}{\xi\left(1+K_{\mathrm{eq}}\right)-\left(1+(1-\xi) \sum_{j=0}^{i-2}\left(\frac{\zeta}{\xi}\right)^{i-j-1}\right)}\right\}\right\},
\end{aligned}
$$

whereas combination of Eq. (49) sequentially yields in turn:

$$
\begin{aligned}
& t_{\mathrm{spn}, \mathrm{tot}, N, \mathrm{ser}}^{*}=\sum_{i=1}^{N} t_{\mathrm{spn}, i}^{*} \\
&=\Omega\left\{\frac{(1-\zeta)(1-\xi)}{\xi}\left(\frac{\xi-\xi^{N+1}}{1-\xi}+\sum_{i=1}^{N} \sum_{j=0}^{i-2} \xi^{i}\left(\frac{\zeta}{\xi}\right)^{i-j-1}\right)\right. \\
&\left.+\frac{\xi-\xi^{N+1}}{1-\xi} \ln \left\{\frac{1}{\zeta}\right\}\right\} .
\end{aligned}
$$

The dimensionless molar rate of production of $\mathrm{P}$ in a series combination of rector/separator sets is then given by:

$r_{\mathrm{P}, \mathrm{ser}}^{*}=\frac{\bar{n}_{\mathrm{P}, \text { tot }, N, \mathrm{ser}}^{*}}{t_{\mathrm{rxn}, \mathrm{tot}, N, \mathrm{ser}}^{*}+t_{\mathrm{spn}, \mathrm{tot}, N, \mathrm{ser}}^{*}}$,

which can then be duly combined with Eqs. (47), (50) and (51), to yield:

$$
r_{\mathrm{P}, \text { ser }}^{*}=\frac{(1-\zeta)\left\{\left(1-\xi^{N}\right)+(1-\xi) \sum_{i=1}^{N} \sum_{j=0}^{i-2}\left(\zeta^{i-j-1} \xi^{j}\right)\right\}}{\left\{\frac{K_{\mathrm{eq}}}{1+K_{\mathrm{eq}}} \ln \left\{\prod_{i=1}^{N}\left(\frac{K_{\mathrm{eq}}-(1-\xi) \sum_{j=0}^{i-2}\left(\frac{\xi}{\xi}\right)^{i-j-1}}{\xi\left(1+K_{\mathrm{eq}}\right)-\left(1+(1-\xi) \sum_{j=0}^{i-2}\left(\frac{\xi}{\xi}\right)^{i-j-1}\right)}\right)\right\}+\Omega\left\{\frac{(1-\zeta)(1-\xi)}{\xi}\left(\frac{\xi-\xi^{N+1}}{1-\xi}+\sum_{i=1}^{N} \sum_{j=0}^{i-2} \xi^{i}\left(\frac{\zeta}{\xi}\right)^{i-j-1}\right)+\frac{\xi-\xi^{N+1}}{1-\xi} \ln \left\{\frac{1}{\zeta}\right\}\right\}\right\}} .
$$


If $\zeta=\xi$, then Eq. (53) can be simplified to:

$$
r_{\mathrm{P}, \mathrm{ser}}^{*}=\frac{(1-\xi)\left\{\left(1-\xi^{N}\right)+(1-\xi) \sum_{i=1}^{N} \sum_{j=0}^{i-2} \xi^{i-1}\right\}}{\left\{\frac{K_{\mathrm{eq}}}{1+K_{\mathrm{eq}}} \ln \left\{\prod_{i=1}^{N}\left\{\frac{K_{\mathrm{eq}}-(i-1)(1-\xi)}{\xi\left(1+K_{\mathrm{eq}}\right)-(1+(1-\xi)(i-1)]}\right\}\right\}+\Omega\left\{\frac{(1-\xi)^{2}}{\xi}\left(\frac{\xi-\xi^{N+1}}{1-\xi}+\sum_{i=1}^{N} \sum_{j=0}^{i-2} \xi^{i}\right)+\frac{\xi-\xi^{N+1}}{1-\xi} \ln \left\{\frac{1}{\xi}\right\}\right\}\right\}},
$$

which, taking advantage from the properties of the sum- By a similar token, combination of Eqs. (35), (37) and (41) mation of the terms of a geometrical series [35] will, after yields:

mathematical rearrangement, yield:

$$
r_{\mathrm{P}, \mathrm{ser}}^{*}=\frac{1+\xi^{N}[N \xi-(1+N)]}{\left\{\frac{K_{\mathrm{eq}}}{1+K_{\mathrm{eq}}} \ln \left\{\prod_{i=1}^{N}\left(\frac{K_{\mathrm{eq}}-(i-1)(1-\xi)}{\xi\left(1+K_{\mathrm{eq}}\right)-[1+(i-1)(1-\xi)]}\right)\right\}+\Omega\left(1+\xi^{N}(N \xi-(1+N))-\frac{\xi-\xi^{N+1}}{1-\xi} \ln \{\xi\}\right)\right\}}
$$

\section{2}

\section{Parallel combination}

In the case of a parallel combination of reactor/separator sets (Fig. 2), and recalling the generic heuristic rule that the best performance is obtained when the inlet stream is equally divided by the similar reactor/separator sets available [36], then application of Eqs. (35), (37) and (38) gives:

$$
\begin{aligned}
\bar{n}_{\mathrm{P}, \mathrm{tot}, \mathrm{par}}^{*} & =\sum_{i=1}^{N} \frac{\bar{n}_{\mathrm{P}, 1}^{*}}{N} \\
& =\sum_{i=1}^{N} \frac{(1-\zeta)(1-\xi)}{N}=(1-\zeta)(1-\xi),
\end{aligned}
$$

where subscript par refers to the parallel combination and subscript tot refers to all reactor/separator sets at $i=1$. On the other hand, the time of reaction process can be obtained via combination of Eqs. (35), (37) and (40), viz.:

$$
t_{\mathrm{rxm}, \mathrm{par}}^{*}=\frac{K_{\mathrm{eq}}}{1+K_{\mathrm{eq}}} \ln \left\{\frac{K_{\mathrm{eq}}}{\left(1+K_{\mathrm{eq}}\right) \xi-1}\right\} \text {. }
$$

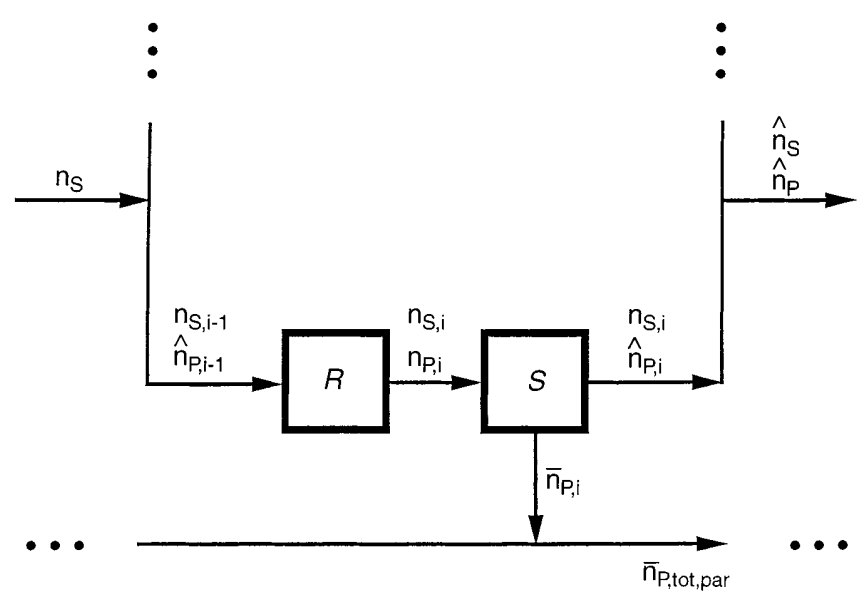

Fig. 2. Schematic representation of the generic $i$-th set, constituted by one reaction (R) and one separation unit (S), in a cascade of $N$ sets in parallel

$$
t_{\text {spn,par }}^{*}=\frac{\Omega}{N}((1-\xi)(1-\zeta)-\xi \ln \{\zeta\}) .
$$

The dimensionless molar rate of production of $\mathrm{P}$ by parallel combinations of reactor/separator sets is then given by:

$$
r_{\mathrm{P}, \mathrm{par}}^{*}=\frac{\bar{n}_{\mathrm{P}, \mathrm{tot}, \mathrm{par}}^{*}}{t_{\mathrm{rxn}, \mathrm{par}}^{*}+t_{\mathrm{spn}, \mathrm{par}}^{*}}
$$

which can then be duly combined with Eqs. (56), (57) and (58) to yield:

$$
\begin{aligned}
& r_{\mathrm{P}, \mathrm{par}}^{*}= \\
& \frac{(1-\zeta)(1-\xi)}{\frac{K_{\mathrm{eq}}}{1+K_{\mathrm{eq}}} \ln \left\{\frac{K_{\mathrm{eq}}}{\left(1+K_{\mathrm{eq}}\right) \xi-1}\right\}+\frac{\Omega}{N}((1-\zeta)(1-\xi)-\xi \ln \{\zeta\})}
\end{aligned}
$$

If $\zeta=\xi$, then Eq. (60) can then be simplified to:

$$
r_{\mathrm{P}, \mathrm{par}}^{*}=\frac{(1-\xi)^{2}}{\frac{K_{\mathrm{eq}}}{1+K_{\mathrm{eq}}} \ln \left\{\frac{K_{\mathrm{eq}}}{\left(1+K_{\mathrm{eq}}\right) \xi-1}\right\}+\frac{\Omega}{N}\left((1-\xi)^{2}-\xi \ln \{\xi\}\right)} .
$$

Plots of the fractional amount of product recovered $\left(\bar{n}_{P}^{*}\right)$, dimensionless total time $\left(t_{\mathrm{rxn}}^{*}+t_{\mathrm{spn}}^{*}\right)$ and dimensionless rate of product recovered $\left(r_{\mathrm{p}}^{*}\right)$ are available as Fig. $3 \mathrm{a}, \mathrm{b}$ and $\mathrm{c}$, respectively, as a function of the number of stages $(N)$, for both situations of series and parallel combination.

\section{3}

\section{Integrated configuration}

Consider now a fully integrated unit, where reaction and separation are carried out simultaneously (Fig. 4) and where again the assumption is made that no product is present at the inlet stream to the system. In this situation, two phases will coexist inside the integrated unit: (i) one phase where the biochemical reaction depicted in Eq. (1) takes place; and (ii) another phase, composed by pure $\mathrm{P}$ which is produced in the reaction and transferred to it. Pure product, $\bar{n}_{\mathrm{p}}^{*}$, will then leave the unit in the upper 

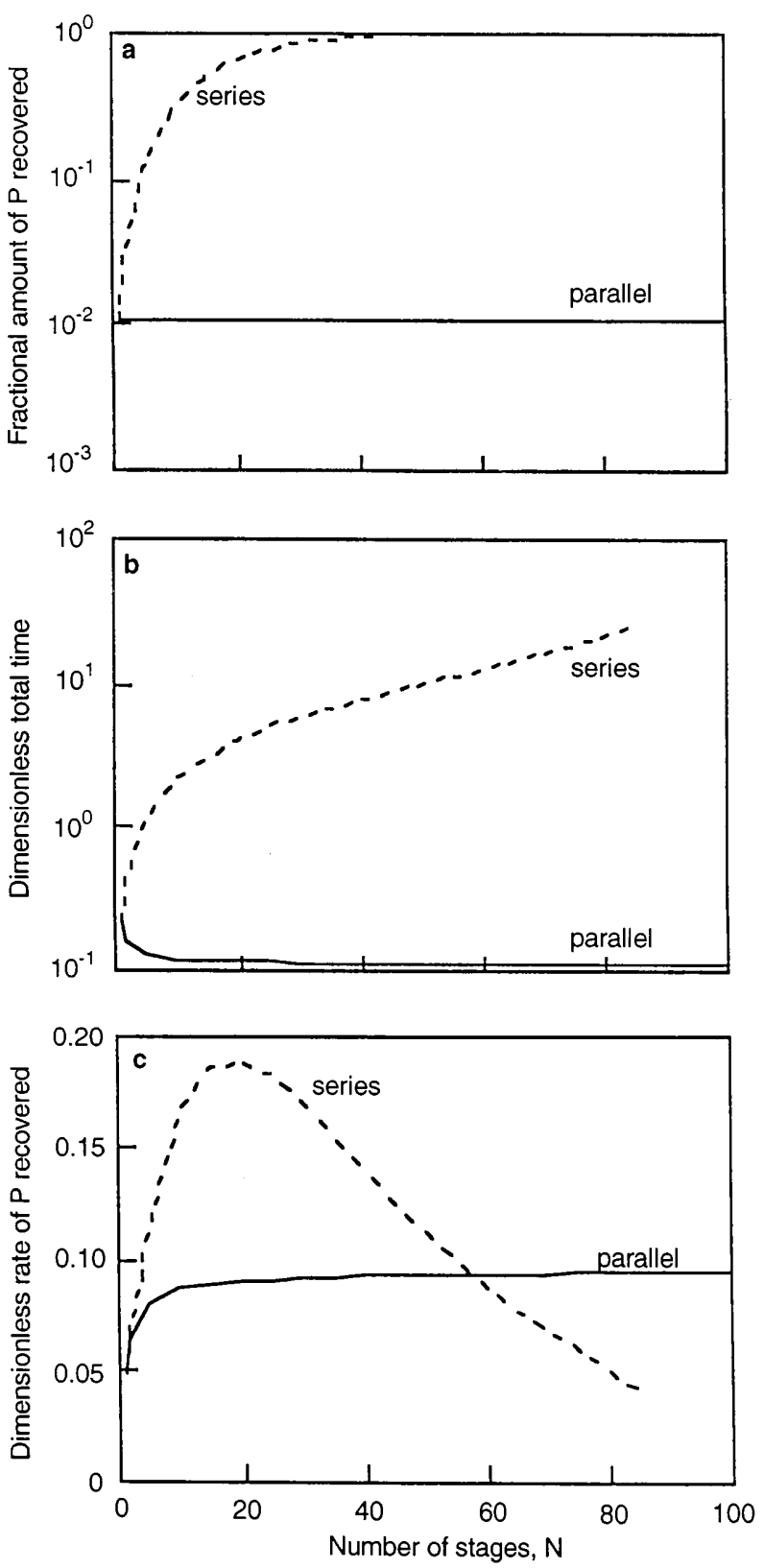

Fig. 3. Variation of a the fractional amount of $\mathrm{P}$ recovered, $\bar{n}_{\mathrm{P}}^{*}$, b the total dimensionless time, $t_{\mathrm{rxn}, \text { tot } N}^{*}+t_{\mathrm{spn}, \mathrm{tot}, N}^{*}$, and $\mathbf{c}$ the dimensionless rate of $\mathrm{P}$ recovered, $r_{\mathrm{P}}^{*}$, for the cases of series combination and parallel combination, as a function of the number of stages, $N$, for $K_{\mathrm{eq}}=10, \zeta=0.9, \xi=0.9$ and $\Omega=1$

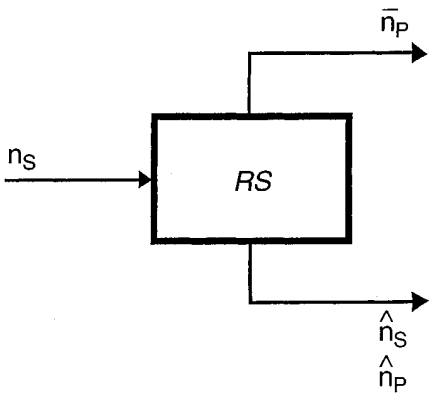

Fig. 4. Schematic representation of an integrated reaction/separation unit stream, whereas unreacted substrate and the remaining product will remain inside the unit and will afterwards be unloaded as $\hat{n}_{\mathrm{S}}$ and $\hat{n}_{\mathrm{P}}$.

A molar balance to both components $\mathrm{P}$ and $\mathrm{R}$ in the lower phase will therefore read:

$\frac{\mathrm{d} n_{\mathrm{S}}}{\mathrm{d} t}=-k_{1} C_{\mathrm{S}} V_{\mathrm{L}}+k_{-1} C_{\mathrm{p}} V_{\mathrm{L}}$,

$\frac{\mathrm{d} n_{\mathrm{P}}}{\mathrm{d} t}=k_{1} C_{\mathrm{S}} V_{\mathrm{L}}-k_{-1} C_{\mathrm{p}} V_{\mathrm{L}}-k_{\mathrm{mt}} A C_{\mathrm{P}}$,

respectively, where $V_{\mathrm{L}}$ denotes the volume of the lower phase which, owing to transfer of $\mathrm{P}$ into the upper phase, varies according to:

$V_{\mathrm{L}}=v_{\mathrm{S}} n_{\mathrm{S}}+v_{\mathrm{P}} n_{\mathrm{P}}$

where $v_{\mathrm{S}}$ and $v_{\mathrm{P}}$ denote the molar volume of substrate and product, respectively, and which, for a matter of simplicity, will be assumed to be equal to one another (i.e.

$v=v_{\mathrm{S}}=v_{\mathrm{P}}$ )

Taking into consideration Eqs. (6), (21), (22), (23) and (34), Eqs. (62) and (63) will, after mathematical rearrangement, yield:

$\frac{\mathrm{d} n_{\mathrm{S}}^{*}}{\mathrm{~d} t^{*}}=\frac{n_{\mathrm{P}}^{*}}{K_{\mathrm{eq}}}-n_{\mathrm{S}}^{*}$,

$\frac{\mathrm{d} n_{\mathrm{P}}^{*}}{\mathrm{~d} t}=n_{\mathrm{S}}^{*}-\frac{n_{\mathrm{P}}^{*}}{K_{\mathrm{eq}}}-\frac{1}{\Omega} \frac{n_{\mathrm{P}}^{*}}{n_{\mathrm{P}}^{*}+n_{\mathrm{S}}^{*}}$,

respectively, where:

$n_{\mathrm{P}}^{*}=1-n_{\mathrm{S}}^{*}$,

$t^{*}=0, n_{\mathrm{S}}^{*}=1, n_{\mathrm{P}}^{*}=0$.

In order to calculate the time taken by the integrated process, mathematical integration of Eqs. (65) and (66) can proceed numerically making use of finite differences. The plot of the dimensionless number of moles of pure $\mathrm{P}$ obtained $\left(\bar{n}_{\mathrm{P}}^{*}\right)$ as a function of (dimensionless) time $\left(t^{*}\right)$ is available as Fig. 5. In order to allow easier understanding,

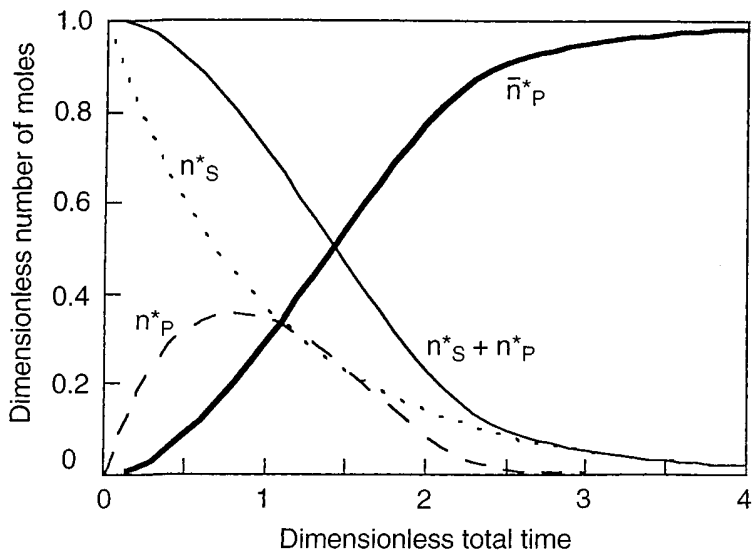

Fig. 5. Variation of the dimensionless number of moles of substrate $\left(n_{\mathrm{S}}^{*}\right)$, of product $\left(n_{\mathrm{P}}^{*}\right)$, and of the dimensionless total number of moles $\left(n_{\mathrm{S}}^{*}+n_{\mathrm{P}}^{*}\right)$ in the lower phase, and of the fractional amount of $\mathrm{P}$ recovered $\left(\bar{n}_{\mathrm{P}}^{*}\right)$ in the upper phase in the case of full integration, as a function of the dimensionless time $\left(t^{*}\right)$ 
the variation of $n_{\mathrm{S}}^{*}, n_{\mathrm{P}}^{*}$ and total number of moles in the lower phase $\left(n_{\mathrm{S}}^{*}+n_{\mathrm{P}}^{*}\right)$ are also represented in this figure.

\section{3}

\section{Discussion}

The aim of this work was to evaluate and compare: (i) the performance of a cascade of $N$ reactor/separator sets placed in a sequential fashion with that of the same cascade of $N$ reactor/separator sets but in parallel, and (ii) the relative improvements eventually achieved by integrating reaction and separation in one single unit. In order for this comparison to be possible, the three systems analysed were considered to work under the same conditions, i.e. no product was considered to be present at the inlet stream $\left(n_{\mathrm{P}, 0}^{*}=0\right.$ and $\left.n_{\mathrm{S}, 0}^{*}=1\right)$, the same 1:1 first order reaction was considered to take place, all reactors and separators were considered to be perfectly similar to one another (thus providing the same results both in terms of conversion and separation), the mixture of substrate and product was assumed to behave ideally from a thermodynamic point of view, and pure product $\mathrm{P}$ was assumed to form the upper phase in either the separator (in the series and parallel configuration) or the integrated unit.

In the theoretical development presented above, five parameters were invoked in order to predict the behaviour of the systems considered:

(i) one cascading parameter, which is the number $(N)$ of reactor/separator sets $(N=1$ for the unit operation process; $N \geq 2$ for the multiple unit, cascaded process); as $N$ tends to infinity, the cascaded process approaches a fully integrated reaction/separation process;

(ii) two reactional parameters, which describe the thermodynamic inhibition (equilibrium constant, $K_{\text {eq }}$ ) and the extent of depletion by chemical reaction $(\xi)$, which, in practical terms, represents the fraction of nonconverted substrate in each reactor, i.e. $\xi=1-\chi$ (where $\chi$ represents the substrate conversion in each reactor unit); and

(iii) two separational parameters, which describe the extent of depletion by separation $(\zeta)$, which, in practical terms, represents the fraction of product which was not recovered in the $\bar{n}_{\mathrm{P}}^{*}$ stream as pure product $\left(\zeta=1-\frac{\bar{n}_{\mathrm{P}}}{n_{\mathrm{p}}}\right)$, and the rate of separation $(\Omega)$, which can be viewed as the ratio of two time scales, i.e. the time scale associated with molecular transport of $\mathrm{P}\left(v n_{\mathrm{S}, 0} / k_{\mathrm{mt}} A\right)$ and the time scale associated with chemical reaction $\left(1 / k_{1}\right)$.

The evaluation of performance of the two types of cascading (Figs. 1 and 2) was made in terms of the fractional amount of product recovered $\left(\bar{n}_{\mathrm{P}, \text { tot, } N}^{*}\right)$, dimensionless total time required $\left(t_{\mathrm{rxn}, \text { tot }, N}^{*}+t_{\mathrm{spn}, \text { tot }, N}^{*}\right)$ and dimensionless rate of product recovered $\left(r_{\mathrm{P}}^{*}\right)$ as a function of the number of reactor/separator sets used $(N)$, either in series or in parallel (Fig. 3a, b and c).

From inspection of Fig. 3a it can be concluded that, in the case of the parallel combination, increasing the number of sets has no influence in the amount of pure product recovered. Such behaviour is expected because the whole modelling was made under the assumption that the inlet stream $\left(n_{S}\right)$ is equally divided by the number of sets available and that those sets are perfectly similar to one another both in terms of conversion and separation. Therefore, if only $\bar{n}_{\mathrm{P}, \text { tot }, N}^{*}$ were to be considered, increasing the number of sets in parallel would only lead to higher equipment costs since, notwithstanding the fact that the degree of recovery of product would be lower in each set, the total amount of product recovered would be exactly the same owing to lumping of the outlet streams. Also, from inspection of Fig. 3b, one can see that the total time taken to achieve the aforementioned degree of recovery of product decreases (this decrease is more apparent for values of $N$ of ca. 10). Such behaviour can be explained by the fact that, if the same degree of conversion and recovery by separation were achieved simultaneously in all reactors and separators placed in parallel, the higher the number of sets the lower the time required for the overall degree of recovery be attained.

A similar analysis for the series combination, based on inspection of Fig. 3a, leads one to the conclusion that the higher the number of sets $(N)$, the higher the amount of product recovered; for a situation characterized by $K_{\text {eq }}=10, \zeta=0.9, \xi=0.9, \Omega=1$ and an $N$ of ca. 30 , virtually all product can be recovered $\left(\bar{n}_{\mathrm{P}, \text { tot, } N}^{*} \approx 1\right)$; remember that, for the same values, the amount of recovery in the parallel combination is ca. one hundred times lower. In the case of the series combination, such behaviour can be explained by the fact that, since pure product is continuously removed when passing from one set to the next, the forward reaction is favoured, so higher amounts of $P$ are produced when compared to what would happen if no product were removed. The increase in the fractional amount of $\mathrm{P}$ recovered is more striking for lower values of $N$ due to the considerable amount of substrate $\mathrm{S}$ still present. The higher the number of sets the lower the increase in the recovery because, although the extent of depletion by reaction $(\xi)$ is assumed as constant, the low amounts of substrate present in the reactor no longer favour production of $\mathrm{P}$. Such fact is emphasized in Fig. 3c, where the rate of recovery $\left(r_{\mathrm{P}, \mathrm{ser}}^{*}\right)$ is depicted, and where an increase in such rate is recorded until the point in which, in Fig. 3a, a steep increase in $\bar{n}_{\mathrm{P}, \text { tot }, N \text {,ser }}^{*}$ is noted; after this point, the increase in $\bar{n}_{\mathrm{P}, \text { tot }, N \text {,ser }}^{*}$ is much lower, and corresponds to a decrease in $r_{\mathrm{P}, \mathrm{ser}}^{*}$ in Fig. 3c. In terms of the total time $\left(t_{\mathrm{rxn}, \mathrm{tot}, N, \mathrm{ser}}^{*}+t_{\mathrm{spn}, \mathrm{tot}, N, \mathrm{ser}}^{*}\right)$ required to achieve the corresponding recovery, it seems more or less obvious that, since the sets are combined in series, the higher the number of sets the higher the time required. From inspection of Fig. $3 \mathrm{~b}$ the dimensionless time taken is in fact much higher than the one required by the parallel combination, but this apparent disadvantage is overcome by the extra fractional amount of product recovered in series. (It should be emphasized that, given the values of $K_{\text {eq }}, \zeta, \xi$ and $\Omega$ selected in these simulations, application of Eqs. (50) and (53) raise limitations in terms of the maximum number of sets that can be considered because of mathematical constraints associated with use of logarithms; in this particular situation, the maximum number of sets allowed was 85). On the other hand, the higher the number of sets used, the higher the capital costs involved, so a compromise between purity and yield of product recovered, and cost associated therewith, should be taken into account.

Evaluation of performance of the integrated configuration was made in terms of the fractional amount of pure 


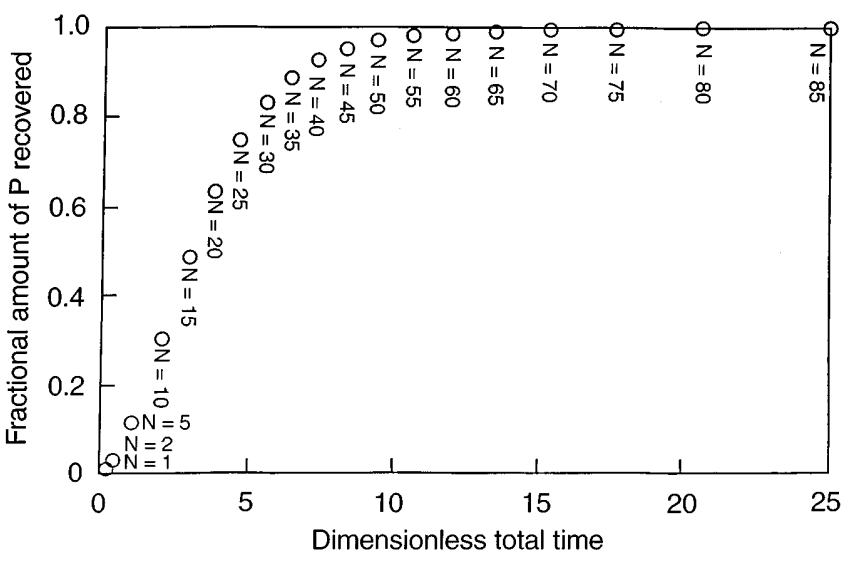

Fig. 6. Total fractional amount of $\mathrm{P}$ recovered, $\bar{n}_{\mathrm{P}, \text { tot,ser }}^{*}$, and dimensionless total time required therefore $\left(t_{\mathrm{rxn}, \text { tot. } N \text {,ser }}^{*}+t_{\mathrm{spn}, \mathrm{tot}, N, \mathrm{ser}}^{*}\right)$ in the case of a series combination of reactor/separator sets, for $K_{\mathrm{eq}}=10, \zeta=0.9, \xi=0.9$ and $\Omega=1$

product recovered as a function of the dimensionless total time taken. Data obtained (values of $K_{\mathrm{eq}}=10$ and $\Omega=1$ were considered for a matter of consistency with the cascading analyses) can be observed in Fig. 5 where, for the sake of easier understanding, variations of the dimensionless number of moles of substrate $\left(n_{\mathrm{S}}^{*}\right)$, product $\left(n_{\mathrm{P}}^{*}\right)$ and total number of moles $\left(n_{\mathrm{S}}^{*}+n_{\mathrm{P}}^{*}\right)$ in the lower phase, as well as the fractional amount of pure product recovered $\left(\bar{n}_{\mathrm{P}}^{*}\right)$ are represented. Inspection of Fig. 5 indicates that the number of moles of substrate in the lower phase $\left(n_{\mathrm{S}}^{*}\right)$ decreases steadily throughout time, thus indicating that conversion of substrate into product is effectively carried out. Product obtained via this reaction either remains in the lower phase $\left(n_{\mathrm{S}}^{*}\right)$ or is transferred to the upper phase for eventual recovery in pure form. Analysis of this plot also shows that the fractional amount of pure product recovered in such integrated system increases steadily, and that for $t^{*}=4$ it approaches unity, hence proving the effectiveness of this configuration.

In order to allow some degree of comparison between the integrated configuration and the series cascaded configuration (which, as seen above, provides better results than the parallel one), the fractional amount of pure product recovered in the series configuration as a function of the dimensionless total time required for each number of sets was plotted in Fig. 6; note that each point represents a different number of sets of reaction/separation units. Comparison between Figs. 5 and 6 shows that, using an integrated system (a single unit where reaction and separation are simultaneously carried out) the dimensionless time taken to attain $\bar{n}_{\mathrm{P}}^{*} \approx 1$ (ideal situation) is ca. 4 . Under the same conditions, and for $t^{*}=4$, the series combination would lead to $\bar{n}_{\mathrm{P}}^{*} \approx 0.625$, which is equivalent to using 20 reactor/separator sets; in fact $\bar{n}_{\mathrm{P}}^{*} \approx 1$ is attained only when ca. 50 sets are used, and corresponds to $t^{*} \approx 10$. This analysis makes it evident that the series combination can provide the same results in terms of recovery of pure product as its integrated counterpart, but only at the expense of much longer time and much higher capital investment, which makes it therefore less attractive.

\section{4}

Taking into consideration the degree of recovery of pure product and the time required to perform such recovery, one concludes that in general (i) series cascading of reactor/separator units provides better results than parallel cascading, and (ii) the integrated approach is more timeand cost-effective than any cascade.

\section{References}

1. Laane, C.; Tramper, J.; Lilly, M.D. (Eds.): Biocatalysis in Organic Media, Amsterdam: Elsevier, 1987

2. Tramper, J.; Vermue, M.H.; Beeftink, H.H.; von Stockar, U. (Eds.): Biocatalysis in Non-conventional Media, Amsterdam: Elsevier, 1992

3. Paiva, A.L.; Malcata, F.X.: Does thermodynamics improve processing when chemical reaction is integrated with physical separation in binary ideal mixtures? Chem. Eng. Sci. 52 (1997) 449-452

4. Paiva, A.L.; Malcata, F.X.: Reversible reaction and diffusion within a porous catalyst slab. Chem. Eng. Sci. 52 (1997) 44294432

5. Paiva, A.L.; Malcata, F.X.: Comparison of the performance of integrated and sequential reaction and separation units in terms of recovery of a desired product. Chem. Eng. Sci. (1999) (in press)

6. Paiva, A.L.; van Rossum, D.; Malcata, F.X.: Cascading reactorseparator sets reduces total time for low yield Michaelis Menten reactions: Model predictions. Biocat. Biotransf. 16 (1998) 205-224

7. Paiva, A.L.; van Rossum, D.; Malcata, F.X.: Integrated vs. sequential reaction and separation: Contributions for a global analysis. Chem. Eng. Sci. 54 (1999) 1825-1836

8. Cabral, J.M.S.: Extractive removal of product by biocatalysis. In: Mattiasson, B. and Holst, O. (Eds.) Extractive Bioconversions, pp. 207-235. New York: Marcel Dekker, 1991

9. Bart, H.J.; Marr, R.; Bauer, U.; Reisinger, H.: Reactive-extraction of L-phenylalanine from an enzymatic solution. In: Sekine, T. (Ed.) Solvent Extraction, pp. 1797-1802. Amsterdam: Elsevier, 1992

10. Roychoudhury, P.K.; Srivastava, A.; Sahai, V.: Extractive bioconversion of lactic acid. In: Fiechter, A. (Ed.) Advances in Biochemical Engineering/Biotechnology, vol. 53, pp. 61-87. Berlin: Springer Verlag, 1995

11. Davies, B.; Jeffrey, G.V.: The continuous trans-esterification of ethyl alcohol and butyl acetate in a sieve plate column. Part III. Trans-esterification in a six plate sieve plate column. Trans Instn. Chem. Engrs. 51 (1973) 275-280

12. Hills, G.A.; Macrae, A.R.; Poulina, R.R.: Ester preparation. Eur. patent $n^{\circ}$. 0383405 (1990)

13. Sundquist, J.; Blanch, H.W.; Wilke, C.R.: Vacuum fermentation. In: Mattiasson, B. and Holst, O. (Eds.) Extractive Bioconversions, pp. 237-258. New York: Marcel Dekker, 1991

14. De Garmo J.L.; Parulekar, V.N.; Pinjala, V.: Consider reactive distillation. Chem. Eng. Prog. 43 (1992) 43-50

15. Paiva, A.L.; Malcata, F.X.: Process integration involving lipase-catalyzed ester synthesis reactions. Biotech. Techn. 8 (1994) 629-634

16. Paiva, A.L.; Malcata, F.X.: Integration of reaction and separation with lipases: Biocatalytic Distillation. In: Malcata, F.X. (Ed.) Engineering of/with Lipases, pp. 597-611. Dordrecht: Kluwer Academic Publishers, 1996

17. Xu, Z.P.; Chuang, K.T.: Kinetics of acetic acid esterification over ion exchange catalysis. Can. J. Chem. Eng. 74 (1996) 493-500

18. Marty, A.; Chulalaksananukul, W.; Willemot, R.M.; Condoret, J.S.: Kinetics of lipase-catalyzed esterification in supercritical $\mathrm{CO}_{2}$. Biotechnol. Bioeng. 39 (1992) 273-280 
19. Marty, A.; Combes, D.; Condoret, J.S.: Continuous reactionseparation processes for enzymatic esterification in supercritical carbon dioxide. Biotechnol. Bioeng. 43 (1994) 497-504

20. Martinek, K.; Klyachko, N.L.; Kabanov, A.V.; Khmelnitsky, Y.L.; Levashov, A.V.: Micellar enzymology: its relation to membranology. Biochim. Biophys. Acta 981 (1989) 161-172

21. Matsumura, M.; Perstraction. In: Mattiasson, B. and Holst, O. (Eds.) Extractive Bioconversions, pp. 91-131. New York: Marcel Dekker, 1991

22. Strathmann, H.; Gudernatsch, W.: Continuous removal of ethanol from bioreactor by pervaporation. In: Mattiasson, B.; Holst, O. (Eds.) Extractive Bioconversions, pp. 67-89. New York: Marcel Dekker, 1991

23. van der Wielen, L.A.M.; Straathof, A.J.J.; Luyben, K.C.A.M.: Adsorptive and chromatographic bioreactors. In: Weijnen, M.P.C.; Drinkenburg, A.A.H. (Eds.) Precision Process Technology, pp. 353-379. Dordrecht: Kluwer, 1993

24. van der Wielen, L.A.M.; Diepen, P.J.; Houwers, J.; Luyben, K.C.A.M.: A countercurrent adsorptive reactor for acidifying bioconversion. Chem. Eng. Sci. 51 (1996) 2315-2325

25. van der Padt, A.; Sewalt, J.J.W.; van't Riet, K.: Membrane bioreactor design to force equilibrium towards a favourable product yield. In: Malcata, F.X. (Ed.) Engineering of/with Lipases, pp. 130-138. Dordrecht: Kluwer, 1996

26. Jansen, M.L.: Integration of ion exchange chromatography with an enzymatic reaction. Ph.D. Thesis, Technical University of Delft, The Netherlands, 1996

27. Jansen, M.L.; van Zessen, E.; Straathof, A.J.J.; van der Wielen, L.A.M.; Luyben, K.C.A.M.; van der Tweel, W.J.J.: Immobili- sation of aminoacylase on an anion exchange column to be used as a chromatographic reactor. Ann. N. Y. Acad. Sci. 799 (1996) 533-540

28. Mazzotti, M.; Kruglov, A.; Neri, B.; Gelosa, D.; Morbidelli, M.: A continuous chromatographic reactor: SMBR. Chem. Eng. Sci. 51 (1996) 1827-1836

29. Takeuchi, K.; Uraguchi, Y.: Experimental studies of a chromatographic moving bed reactor. Catalytic oxidation of carbon monoxide on activated alumina as a model reaction. J. Chem. Eng. Japan, 10 (1977) 455-460

30. Parvaresh, F.; Robert, H.; Thomas, D.; Legoy, M.D.: Gas phase transesterification reactions catalyzed by lipolytic enzymes. Biotechnol. Bioeng. 39 (1992) 467-473

31. Groot, W.J.; Kraayenbrink, M.R.; Waldram, R.H.; van der Lans, R.G.J.M.; Luyben, K.C.A.M.: Ethanol production in an integrated process of fermentation and ethanol recovery by pervaporation. Bioproc. Eng. 8 (1992) 9-11

32. Kemp, R.A.; Macrae, A.R.: Esterification process. Eur. patent $\mathrm{n}^{\circ} .0506159$ (1992)

33. Paiva, A.L.; Malcata, F.X.: Integration of reaction and separation with lipases: an overview. J. Molecular Catalysis B: Enzymatic, 3 (1997) 99-109

34. Segel, I.H.: Enzyme Kinetics - Behavior and Analysis of Rapid Equilibrium and Steady-State Enzyme Systems. New York: Wiley, 1993

35. Malcata, F.X.: CSTRs in biochemical reactions: An optimization problem. Chem. Eng. Ed. 23 (1989) 112-116

36. Hill, C.G.: An Introduction to Chemical Enginering Kinetics and Reactor Design. New York: Wiley, 1977 